\title{
Anisotropic Surface Meshing
}

\author{
Siu-Wing Cheng* $\quad$ Tamal K. Dey ${ }^{\dagger} \quad$ Edgar A. Ramos ${ }^{\ddagger} \quad$ Rephael Wenger $^{\S}$
}

\begin{abstract}
We study the problem of triangulating a smooth closed implicit surface $\Sigma$ endowed with a $2 \mathrm{D}$ metric tensor that varies over $\Sigma$. This is commonly known as the anisotropic surface meshing problem. We extend the 2D metric tensor naturally to $3 \mathrm{D}$ and employ the $3 \mathrm{D}$ anisotropic Voronoi diagram of a set $P$ of samples on $\Sigma$ to triangulate $\Sigma$. We prove that a restricted dual, Mesh $P$, is a valid triangulation homeomorphic to $\Sigma$ under appropriate conditions. We also develop an algorithm for constructing $P$ and Mesh $P$. In addition to being homeomorphic to $\Sigma$, each triangle in Mesh $P$ is well-shaped when measured using the 3D metric tensors of its vertices. Users can set upper bounds on the anisotropic edge lengths and the angles between the surface normals at vertices and the normals of incident triangles (measured both isotropically and anisotropically).
\end{abstract}

\section{Introduction}

Many applications in science and engineering need to mesh a smooth closed surface $\Sigma$ for numerical simulations, prototyping, rendering and various other purposes. A variety of algorithms and software systems based on them have been developed for the problem by the mesh generation, computer graphics, and image processing communities, e.g. [4, 12, 15, 17, 19, 21]. A subset of them focus on implicit surfaces, e.g. $[4,15,17,19]$. An implicit surface representation is appealing for the relative ease in combining surfaces, computing linesurface intersection, and carrying out the inside/outside test. In this representation, there is an implicit function $E: \mathbb{R}^{3} \rightarrow \mathbb{R}$ and $\Sigma$ is its zero-set $E(x)=0$. The surface $\Sigma$ can have arbitrary genus.

Despite the experimental success, it is not until recently that some algorithms have been designed with a guarantee about output quality: specifically, the out-

\footnotetext{
*Supported by the Research Grant Council, Hong Kong, China (HKUST6181/04E). Department of Computer Science, HKUST, Hong Kong. Email: scheng@cs.ust.hk.

†Supported by NSF CCR-0430735 and NSF DMS-0310642. Department of Computer Science and Engineering, Ohio State University, USA. Email: tamaldey@cse.ohio-state.edu.

$\ddagger$ Department of Computer Science, University of Illinois at Urbana-Champaign, USA. Email: eramosn@cs.uiuc.edu.

$\S$ Department of Computer Science and Engineering, Ohio State University, USA. Email: wenger@cse.ohio-state.edu.
}

put triangulation being homeomorphic to the input surface $\Sigma$ and being geometrically close to it. Cheng, Dey, Edelsbrunner, and Sullivan [10] designed a meshing algorithm for skin surfaces in molecular modeling. Boissonnat and Oudot [6] and Cheng, Dey, Ramos, and Ray [11] proposed algorithms for general implicit surfaces. Although both algorithms sample points and compute a restricted Delaunay triangulation, they differ in many ways. In particular, a lower bound estimation of the local feature size is needed in [6] where critical point computations replacing local feature size are required in [11]. Boissonnat, Cohen-Steiner, and Vetger [7] designed an algorithm to approximate the level sets of the implicit function, which yields an isotopic surface triangulation.

The above certified meshing algorithms are designed for the isotropic setting in which nearly equilateral triangles are used. Sometimes, long and skinny triangles offer better approximation because they adapt to the principal curvatures of the surface. They are also preferred in numerical simulations where the physical phenomena are strongly directional. These applications call for an anisotropic triangulation. The general formulation is to associate a 2D metric tensor $M_{x}$, a $2 \times 2$ positive definite symmetric matrix, with each point $x \in \Sigma$. Its eigenvalues $0<k_{x, 1} \leq k_{x, 2}$ and corresponding orthogonal unit eigenvectors $d_{x, 1}$ and $d_{x, 2}$ specify the following deformation on the tangent plane at $x$. Any point $z$ on the tangent plane is mapped to the point $x+\left\langle z-x, d_{x, 1}\right\rangle \cdot \sqrt{k_{x, 1}} d_{x, 1}+\left\langle z-x, d_{x, 2}\right\rangle \cdot \sqrt{k_{x, 2}} d_{x, 2}$. So the distance components along $d_{x, 1}$ and $d_{x, 2}$ are scaled by factors $\sqrt{k_{x, 1}}$ and $\sqrt{k_{x, 2}}$, respectively. We call $\sqrt{k_{x, 1}}$ and $\sqrt{k_{x, 2}}$ the scaling factors and $d_{x, 1}$ and $d_{x, 2}$ the principal directions. The points on the tangent plane at distance 1 from $x$ (measured using $M_{x}$ ) lie on an ellipse centered at $x$ with $d_{x, 1}$ as the major axis. To adapt to $M_{x}$, the surface mesh triangles near $x$ should be elongated in direction $d_{x, 1}$.

Few theoretical results are known for the anisotropic triangulation problem. Algorithms were obtained recently for the problem of computing a $2 \mathrm{D}$ conformal anisotropic triangulation of a planar straight line graph with no angle much less $\pi / 2[8,16]$. The definition of anisotropic Voronoi diagram originates from the work of Labelle and Shewchuk [16]. A surface associated with 
a varying metric tensor is a Riemannian manifold. Leibon and Letscher [18] showed that if the sampling density is sufficiently high, the dual of the Voronoi diagram defined using the shortest path distance is a valid triangulation. Unfortunately, this distance calculation is computationally difficult and it is unclear how to obtain the samples in the first place.

In this paper, we assume that the largest scaling factor at any point $x \in \Sigma$ is bounded from above, but we do not need to know the bound. We assume an oracle to return $M_{x}$ for each point $x \in \Sigma$, but we do not need a closed form formula for generating $M_{x}$. The metric tensor should vary smoothly and we will make this concrete later. We extend $M_{x}$ to a $3 \mathrm{D}$ metric tensor by adding a distance component with scaling factor $\sqrt{k_{x, 2}}$ in the normal direction at $x$. On planes parallel to the tangent plane at $x$, the 3D metric tensor measures angles and lengths identically as $M_{x}$. Then we employ the 3D anisotropic Voronoi diagram of a set $P$ of samples on $\Sigma$ to triangulate $\Sigma$. It is known that the dual of a $3 \mathrm{D}$ anisotropic Voronoi diagram may not be a valid tetrahedralization $[8,16]$. However, we show that a restricted dual, Mesh $P$, is a valid triangulation homeomorphic to $\Sigma$ under appropriate conditions. (See Theorem 5.1.)

We develop an incremental point insertion algorithm to grow $P$ as in the isotropic case [11]. The final Mesh $P$ is homeomorphic to $\Sigma$. Users can specify two upper bounds $\ell$ and $\zeta$ such that the edge length is at most $\ell$ when measured using the 3D metric tensors of the edge endpoints, and the surface normal at a vertex makes an angle at most $\zeta$ with the normal of any incident triangle. The bound $\zeta$ also holds when the angle is measured using the 3D metric tensor of the vertex. Each triangle in Mesh $P$ has bounded aspect ratio when measured using the 3D metric tensors of its vertices. When $\zeta$ is small, a triangle will be almost parallel to the tangent planes at its vertices. Then bounded aspect ratio in the anisotropic setting implies that for each triangle vertex $x$, the triangle is elongated more or less in the direction $d_{x, 1}$ as desired.

\section{Metric extension and preliminaries}

Vector and matrix. We use $\|\cdot\|$ to denote the $l^{2}$-norm and the matrix 2-norm. So $\left\|(a, b, c)^{t}\right\|=\sqrt{a^{2}+b^{2}+c^{2}}$. For a real symmetric matrix $A$, take the absolute values of its eigenvalues and let $\lambda_{\max }$ and $\lambda_{\min }$ be the largest and smallest ones, respectively. Then $\|A\|=\lambda_{\max }$ and $\left\|A^{-1}\right\|=1 / \lambda_{\min }$.

3D metric tensor. At each point $x \in \Sigma$, we define a 3D metric tensor $K_{x}=U_{x}^{t} \cdot D_{x} \cdot U_{x}$, where $D_{x}$ is a $3 \times 3$ diagonal matrix with entries $k_{x, 1}, k_{x, 2}$, and $k_{x, 3}$ from top left to bottom right. The matrix $U_{x}$ is a $3 \times 3$ matrix and it transforms space so that the principal directions and the unit outward normal at $x$ become the coordinate system. The new entry $k_{x, 3}$ should be chosen so that $k_{x, 3} \geq k_{x, 2}$. A natural choice is $k_{x, 3}=k_{x, 2}$ and this corresponds to inflating the "unit ellipse" on the tangent plane at $x$ into a "unit ellipsoid" in 3D. If desired, one can also choose $k_{x, 3}$ to be greater than $k_{x, 2}$. Our results hold as long as $\max _{x \in \Sigma} k_{x, 3}$ is bounded from above. One can express $K_{x}=F_{x}^{t} \cdot F_{x}$, where $F_{x}=U_{x}^{t} \cdot D_{x}^{\prime} \cdot U_{x}$ and the diagonal entries of $D_{x}^{\prime}$ are $\sqrt{k_{x, 1}}, \sqrt{k_{x, 2}}$, and $\sqrt{k_{x, 3}}$ from top left to bottom right. From the perspective of $x$, the distance between two points $a, b \in \mathbb{R}^{3}$ is $\left\|F_{x} \cdot(b-a)\right\|$.

3D Linear map. For any point $x \in \Sigma$, we define a map $\varphi_{x}$ such that for any point $b \in \mathbb{R}^{3}, \varphi_{x}(b)=F_{x} \cdot b$. For any subset $B \subseteq \mathbb{R}^{3}$, we use $\varphi_{x}(B)$ to denote $\left\{\varphi_{x}(b)\right.$ : $b \in B\}$. We call $\mathbb{R}^{3}$ the primal space and $\varphi_{x}\left(\mathbb{R}^{3}\right)$ the $\varphi_{x}$-space. We extend the notation $\varphi_{x}(\cdot)$ in several ways to ease the presentation. If we write a vector $d \in \mathbb{R}^{3}$ as a triple of real numbers, then $F_{x} \cdot d$ is the vector in the $\varphi_{x}$ space that $d$ is mapped to. We use $\varphi_{x}(d)$ to denote $F_{x} \cdot d$. Given two vectors $d_{1}$ and $d_{2}$, we use $\angle d_{1}, d_{2}$ to denote the angle between $d_{1}$ and $d_{2}$ and we use $\varphi_{x}\left(\angle d_{1}, d_{2}\right)$ to denote $\angle \varphi_{x}\left(d_{1}\right), \varphi_{x}\left(d_{2}\right)$. Given three points $a, b, c$, we use $\angle a b c$ to denote $\angle a-b, c-b$. Given two points $a, b \in \mathbb{R}^{3}$, we use $d_{x}(a, b)$ to denote the distance $\left\|\varphi_{x}(a)-\varphi_{x}(b)\right\|$ in the $\varphi_{x}$-space. In the $\varphi_{x}$-space, $x$ 's distance metric becomes isotropic. If $y$ 's distance metric is nearly isotropic in the $\varphi_{x}$-space, the bisector surface of $\varphi_{x}(x)$ and $\varphi_{x}(y)$ intersects the segment $\varphi_{x}(x y)$ at nearly right angle and the bisector surface is nearly perpendicular to $\varphi_{x}(x y)$ near the intersection point. Collinearity and incidence are preserved by $\varphi_{x}$. If a plane $H$ is tangent to $\Sigma, \varphi_{x}(H)$ is also tangent to $\varphi_{x}(\Sigma)$. Angles are not preserved in general. But if $d_{1}$ is a normal vector at $x$ and $d_{2}$ is orthogonal to $d_{1}$, then $\varphi_{x}\left(d_{1}\right)$ and $\varphi_{x}\left(d_{2}\right)$ are also orthogonal.

Propagating the metric. The medial axis $\mathcal{X}$ of $\Sigma$ is the set of centers of maximal balls empty of points on $\Sigma$. For any point $x \in \Sigma$, the local feature size $f(x)$ is the distance from $x$ to $\mathcal{X}$. For any point $p \in \mathbb{R}^{3} \backslash \mathcal{X}$, we use $\tilde{p}$ to denote the closet point on $\Sigma$ to $p$. Define $\Sigma^{*}$ to be the set of points $p$ whose distance from $\Sigma$ is at most $f(\tilde{p}) / 2$. For each $p \in \Sigma^{*}$, define $F_{p}=F_{\tilde{p}}$ and $K_{p}=K_{\tilde{p}}$. The set of points $\Sigma^{*} \backslash \Sigma$ and their matrices are only needed for analysis and our algorithm never computes them.

Distortion. The relative distortion between two points $p, q \in \Sigma^{*}$ is $\tau_{p q}=\max \left\{\left\|F_{p} \cdot F_{q}^{-1}\right\|,\left\|F_{q} \cdot F_{p}^{-1}\right\|\right\}$. We have $d_{p}(a, b)=\left\|F_{p} \cdot(a-b)\right\| \leq\left\|F_{p} \cdot F_{q}^{-1}\right\| \cdot d_{q}(a, b) \leq$ $\tau_{p q} d_{q}(a, b)$. Conversely, we also have $d_{p}(a, b) \geq$ 
$d_{q}(a, b) / \tau_{p q}$. Define $\tau_{*}=(65 / 64)^{1 / 4}$. For each point $p \in$ $\Sigma^{*}$, let $\gamma(p)$ denote $\max \left\{\lambda \in \mathbb{R}: \forall q \in \Sigma^{*}, d_{p}(p, q) \leq\right.$ $\left.\lambda \Rightarrow \tau_{p q} \leq \tau_{*}\right\}$. We assume that $F_{x}$ varies smoothly over $x \in \Sigma$ so that $\min _{p \in \Sigma^{*}} \gamma(p)$ is strictly positive. For any points $x, y \in \Sigma$, we define $f_{x}(y)$ to be the distance from $\varphi_{x}(y)$ to the medial axis of $\varphi_{x}(\Sigma)$ in the $\varphi_{x}$-space. Define $\gamma=\min \left\{\min _{p \in \Sigma^{*}} \gamma(p), \min _{x \in \Sigma} f_{x}(x)\right\}$. Thus if $d_{x}(x, y) \leq \gamma$, then $\tau_{x y} \leq \tau_{*}$. The value $\gamma$ defines a neighborhood of $x$ in which the geometry and the metric tensor do not vary much. The value $\gamma$ is only needed for analysis and our algorithm never computes it.

Anisotropic Voronoi diagram. Let $P$ be a finite set of points on $\Sigma$. The anisotropic Voronoi cell of $u \in P$ is $V_{u}(P)=\left\{p \in \mathbb{R}^{3}: \forall v \in P, d_{u}(p, u) \leq d_{v}(p, v)\right\}$. For $0 \leq j \leq 3$, the closed faces shared by $4-j$ Voronoi cells are called $j$-dimensional Voronoi faces. We denote a 2 dimensional Voronoi facet $V_{u}(P) \cap V_{v}(P)$ by $V_{u v}(P)$ and a 1-dimensional Voronoi edge $V_{u}(P) \cap V_{v}(P) \cap V_{w}(P)$ by $V_{u v w}(P)$. The anisotropic Voronoi diagram Vor $P$ is the collection of Voronoi cells together with their boundary faces. The diagram Vor $P$ is the lower envelope of $|P|$ surfaces in $\mathbb{R}^{4}$. It has $O\left(|P|^{4+\epsilon}\right)$ complexity [20]. The restricted dual, Mesh $P$, is a simplicial complex comprising of the points in $P$, the edges $u v$ such that $V_{u v}(P) \cap \Sigma \neq \emptyset$, and the triangles uvw such that $V_{u v w}(P) \cap \Sigma \neq \emptyset$.

Wedge and visibility. Let $Q$ be a set of points on $\Sigma$. Define the wedge of $Q$ to be wedge $(Q)=\left\{x \in \mathbb{R}^{3}\right.$ : $\left.\varphi_{v_{i}}\left(\angle x v_{i} v_{j}\right)<\pi / 2, \quad \forall v_{i}, v_{j} \in Q\right\}$. By definition, wedge $(Q)$ is an open set.

Lemma 2.1. Let $\tau>1$ be a constant. Let $v_{1}$ and $v_{2}$ be points on $\Sigma$. Let $x$ be a point in $V_{v_{1} v_{2}}\left(\left\{v_{1}, v_{2}\right\}\right)$.

(i) If $x \in$ wedge $\left(\left\{v_{1}, v_{2}\right\}\right)$, the interior of the segment $v_{i} x$ lies strictly inside $V_{v_{i}}\left(\left\{v_{1}, v_{2}\right\}\right)$.

(ii) If $x \notin$ wedge $\left(\left\{v_{1}, v_{2}\right\}\right)$ and $\tau_{v_{1} v_{2}} \leq \tau$, then $d_{v_{i}}\left(v_{i}, x\right) \geq \frac{1}{\sqrt{\tau^{2}-1}} \cdot \min \left\{d_{v_{1}}\left(v_{1}, v_{2}\right), d_{v_{2}}\left(v_{1}, v_{2}\right)\right\}$ for $1 \leq i \leq 2$.

(iii) Let $Q$ be a set of points on $\Sigma$ such that $\tau_{v w} \leq \tau$ for any $v, w \in Q$. If the segment vp does not lie inside $V_{v}(Q)$ for some point $p$ on the boundary of $V_{v}(Q)$, then $d_{v}(v, p) \geq \frac{1}{\sqrt{\tau^{2}-1}} \cdot \min \left\{d_{v}(v, w), d_{w}(v, w)\right\}$ for some $w \in Q$.

Proof. (i) and (ii) have been proved in [16]. Consider (iii). As we walk from $v$ towards $p$ along $v p$, we must leave $V_{v}(Q)$ and then reenter $V_{v}(Q)$ at some point $q$. The point $q$ lies on $V_{v w}(Q)$ for some $w \in Q$, and $v q$ does not lie inside $V_{v}(\{v, w\})$. By (i), $q \notin$ wedge $(\{v, w\})$. Then by (ii), $d_{v}(v, p) \geq d_{v}(v, q) \geq$ $\frac{1}{\sqrt{\tau^{2}-1}} \cdot \min \left\{d_{v}(v, w), d_{w}(v, w)\right\}$.

\section{Surface normal variation}

For any point $v, x \in \Sigma$, we use $\mathbf{n}(x)$ to denote the unit outward normal of $\Sigma$ at $x$ and $\mathbf{n}_{v}(x)$ to denote the unit outward normal of $\varphi_{v}(\Sigma)$ at $\varphi_{v}(x)$. Note that $\mathbf{n}_{x}(x)=\mathbf{n}(x)$ for any point $x \in \Sigma$. The main results in this section, as stated in Lemma 3.2 below, are concerned with the variation of the surface normal. Lemma 3.2(i) states that if two points $v_{1}, v_{2} \in \Sigma$ are very near in the $\varphi_{v_{1}}$-space, the segment $\varphi_{v_{1}}\left(v_{1} v_{2}\right)$ is nearly orthogonal to $\mathbf{n}_{v_{1}}\left(v_{1}\right)$. The first half of Lemma 3.2(ii) states that if $v_{1}$ and $v_{2}$ are very near in the $\varphi_{v}$-space, then $\angle \mathbf{n}_{v}\left(v_{1}\right), \mathbf{n}_{v}\left(v_{2}\right)$ is small. These results are the anisotropic versions of known results in the isotropic setting $[2,10]$. Their proofs are the same too. Lemma 3.2(ii) states further that if $v_{1}$ and $v_{2}$ are very near in the $\varphi_{v_{1}}$-space, then $\angle \mathbf{n}\left(v_{1}\right), \mathbf{n}\left(v_{2}\right)$ is small. This requires transferring the normal deviation in the $\varphi_{v_{1}}$-space to the primal space, although $v_{1}$ and $v_{2}$ may be far apart in the primal space. The following result enables the transfer.

Lemma 3.1. Let $x$ be a point on $\Sigma$. Let $H$ be a plane in the primal space. Let $d_{1}$ denote a vector normal to $H$ that makes an acute angle with $\mathbf{n}(x)$. Let $d_{2}$ denote a vector normal to $\varphi_{x}(H)$ that makes an acute angle with $\mathbf{n}_{x}(x)$. Then $\angle \mathbf{n}(x), d_{1} \leq \angle \mathbf{n}_{x}(x), d_{2}$.

Proof. Orient $\mathbb{R}^{3}$ so that the principal directions and the outward surface normal at $x$ form the coordinate system. Then $\mathbf{n}_{x}(x)=\mathbf{n}(x)=(0,0,1)^{t}$ points vertically upward. By our orientation of $\mathbb{R}^{3}$, $F_{x}$ is a diagonal matrix and its diagonal entries are $\sqrt{k_{x, 1}}, \sqrt{k_{x, 2}}$ and $\sqrt{k_{x, 3}}$ from top left to bottom right. Assume that $d_{2}=(a, b, c)^{t}$. For any vector $u=\left(e_{1}, e_{2}, e_{3}\right)^{t}$ parallel to the plane $H$, $\left\langle d_{2}, F_{x} \cdot u\right\rangle=\sqrt{k_{x, 1}} a e_{1}+\sqrt{k_{x, 2}} b e_{2}+\sqrt{k_{x, 3}} c e_{3}=$ $\left\langle F_{x} \cdot d_{2}, u\right\rangle$. Observe that $F_{x} \cdot u$ is a vector parallel to $\varphi_{x}(H)$. Thus, $\left\langle d_{2}, F_{x} \cdot u\right\rangle=\left\langle F_{x} \cdot d_{2}, u\right\rangle=0$. Since this holds for any vector $u$ parallel to $H$, $F_{x} \cdot d_{2}$ is normal to $H$. Consider $\cos \angle \mathbf{n}_{x}(x), d_{2}=$ $\left\langle\mathbf{n}_{x}(x), d_{2}\right\rangle /\left(\left\|\mathbf{n}_{x}(x)\right\| \cdot\left\|d_{2}\right\|\right)=c / \sqrt{a^{2}+b^{2}+c^{2}}$. Since $\angle \mathbf{n}_{x}(x), d_{2}<\pi / 2$ by assumption, we conclude that $c>0$. If $F_{x} \cdot d_{2}$ and $d_{1}$ have opposite orientations, then $\cos \angle \mathbf{n}(x), d_{1}=\left\langle\mathbf{n}(x),-\left(F_{x} \cdot d_{2}\right)\right\rangle /\left(\|\mathbf{n}(x)\| \cdot\left\|F_{x} \cdot d_{2}\right\|\right)=$ $-c \sqrt{k_{x, 3}} / D$, where $D=\sqrt{k_{x, 1} a^{2}+k_{x, 2} b^{2}+k_{x, 3} c^{2}}$. But then $\cos \angle \mathbf{n}(x), d_{1}$ is negative, contradicting the assumption that $\angle \mathbf{n}(x), d_{1}<\pi / 2$. So $\cos \angle \mathbf{n}(x), d_{1}=$ $c \sqrt{k_{x, 3}} / D \geq c / \sqrt{a^{2}+b^{2}+c^{2}}=\cos \angle \mathbf{n}_{x}(x), d_{2}$. Hence, $\angle \mathbf{n}(x), d_{1} \leq \angle \mathbf{n}_{x}(x), d_{2}$. 
Recall that for any point on $\Sigma$, the value $\gamma$ defines a neighborhood in which the geometry and the metric tensor do not vary much.

Lemma 3.2. Define the function $\alpha(c)=c /(1-4 c)$. Let $v_{1}, v_{2}$ be points in $\Sigma$.

(i) Let $\phi=\angle \varphi_{v_{1}}\left(v_{2}-v_{1}\right), \mathbf{n}_{v_{1}}\left(v_{1}\right)$. Then $d_{v_{1}}\left(v_{1}, v_{2}\right) \geq$ $2 \gamma \cos (\min \{\phi, \pi-\phi\})$.

(ii) Let $v$ be a point in $\Sigma$. If $d_{v}\left(v_{1}, v_{2}\right) \leq c \gamma$ for some constant $c<1 / 4$, then $\angle \mathbf{n}_{v}\left(v_{1}\right), \mathbf{n}_{v}\left(v_{2}\right) \leq \alpha(c)$. If $v=v_{1}$, then $\angle \mathbf{n}\left(v_{1}\right), \mathbf{n}\left(v_{2}\right) \leq \angle \mathbf{n}_{v_{1}}\left(v_{1}\right), \mathbf{n}_{v_{1}}\left(v_{2}\right) \leq$ $\alpha(c)$.

Proof. Applying the original proof in [10] in the $\varphi_{v_{1}}-$ space proves (i). Consider (ii). Applying the original proof in [2] in the $\varphi_{v}$-space bounds $\angle \mathbf{n}_{v}\left(v_{1}\right), \mathbf{n}_{v}\left(v_{2}\right)$. Suppose that $v=v_{1}$. Let $B$ be the ball in the $\varphi_{v_{1}}$-space centered at $\varphi_{v_{1}}\left(v_{1}\right)$ with radius $c \gamma$. Because the radius of $B$ is less than $f_{v_{1}}\left(v_{1}\right)$, it is known that $B \cap \varphi_{v_{1}}(\Sigma)$ is a topological disk [5]. We go back to the primal space. Let $v_{2}$ be a point in $\varphi_{v_{1}}^{-1}(B) \cap \Sigma$. We first show that $\angle \mathbf{n}\left(v_{1}\right), \mathbf{n}\left(v_{2}\right)<\pi / 2$. Assume to the contrary that $\angle \mathbf{n}\left(v_{1}\right), \mathbf{n}\left(v_{2}\right) \geq \pi / 2$. Since $\varphi_{v_{1}}^{-1}(B) \cap \Sigma$ is connected, there is a path from $v_{1}$ to $v_{2}$ in $\varphi_{v_{1}}^{-1}(B) \cap \Sigma$. So there is a point $v_{3} \in \varphi_{v_{1}}^{-1}(B) \cap \Sigma$ on this path such that $\angle \mathbf{n}\left(v_{1}\right), \mathbf{n}\left(v_{3}\right)=\pi / 2$. But then $\mathbf{n}_{v_{1}}\left(v_{3}\right)$ is also orthogonal to $\mathbf{n}_{v_{1}}\left(v_{1}\right)$, contradicting the fact that $\angle \mathbf{n}_{v_{1}}\left(v_{1}\right), \mathbf{n}_{v_{1}}\left(v_{3}\right) \leq \alpha(c)$ as $d_{v_{1}}\left(v_{1}, v_{3}\right) \leq c \gamma$. So we have $\angle \mathbf{n}\left(v_{1}\right), \mathbf{n}\left(v_{2}\right)<\pi / 2$ and $\angle \mathbf{n}_{v_{1}}\left(v_{1}\right), \mathbf{n}_{v_{1}}\left(v_{2}\right) \leq \alpha(c)<\pi / 2$. By applying Lemma 3.1 to $v_{1}$ and the tangent plane at $v_{2}$, we get $\angle \mathbf{n}\left(v_{1}\right), \mathbf{n}\left(v_{2}\right) \leq \angle \mathbf{n}_{v_{1}}\left(v_{1}\right), \mathbf{n}_{v_{1}}\left(v_{2}\right)$.

\section{Triangles}

Let $t=v_{1} v_{2} v_{3}$ be a triangle with vertices on $\Sigma$. We use $\mathbf{n}(t)$ to denote the normal of $t$ in the primal space that makes an acute angle with $\mathbf{n}\left(v_{i}\right)$, where $v_{i}$ is the vertex with the largest angle in $t$. Similarly, for any point $v \in \Sigma, \mathbf{n}_{v}(t)$ denotes the normal of $\varphi_{v}(t)$ that makes an acute angle with $\mathbf{n}_{v}\left(v_{j}\right)$, where $\varphi_{v}\left(v_{j}\right)$ is the vertex with the largest angle in $\varphi_{v}(t)$. We use length $(t)$ to denote $\min _{1 \leq i \leq 3} s_{i}$, where $s_{i}$ is the minimum side length of $\varphi_{v_{i}}(t)$. The main results in this section are that under suitable conditions, $\varphi_{v_{i}}(t)$ is well-shaped (Lemma 4.1), both $\angle \mathbf{n}_{v_{i}}(t), \mathbf{n}_{v_{i}}\left(v_{i}\right)$ and $\angle \mathbf{n}(t), \mathbf{n}\left(v_{i}\right)$ are small (Lemma 4.2), and the dihedral angles between adjacent triangles are close to $\pi$ (Lemma 4.4). The analogous version of Lemma 4.1 in the plane has been proved in [16].

In the isotropic setting, a triangle is well-shaped if its radius-edge ratio (the ratio of circumradius to shortest edge length) is bounded. In our case, the ratio $d_{v_{i}}\left(v_{i}, z\right) /$ length $(t)$, where $z \in V_{t}\left(\left\{v_{1}, v_{2}, v_{3}\right\}\right)$, plays a critical role. Recall that $\tau_{*}$ denotes the constant $(65 / 64)^{1 / 4}$.

Lemma 4.1. Let $\rho \in(1, \sqrt{2}]$ be a constant. Let $t=v_{1} v_{2} v_{3}$ be a triangle with vertices on $\Sigma$. Assume that $d_{v_{i}}\left(v_{i}, z\right) \leq 4 \rho \cdot \operatorname{length}(t)$ for some point $z \in V_{t}\left(\left\{v_{1}, v_{2}, v_{3}\right\}\right)$. Let $v \in \Sigma$ be a point such that $\tau_{v v_{i}} \leq \tau_{*}$ for $1 \leq i \leq 3$. Then for each angle $\phi$ of $t$, $\varphi_{v}(\phi) \geq \sin ^{-1}(\chi)$, where $\chi=\frac{1-32\left(\tau_{*}^{4}-1\right)}{128 \tau_{*}^{2}}$. Hence, the radius-edge ratio of $\varphi_{v}(t)$ is at most $\rho_{1}=2 / \chi$.

Proof. We go to the $\varphi_{v}$-space. Let $a_{i}=\varphi_{v}\left(v_{i}\right)$ for $1 \leq i \leq 3$. Let $y=\varphi_{v}(z)$. Let $x$ be the projection of $y$ onto the plane of $\varphi_{v}(t)$.

Each triangle edge incident to $a_{i}$ bounds an open halfplane disjoint from $\varphi_{v}(t)$. We call the intersection of these two open halfplanes the negative cone of $a_{i}$. We claim that $x$ does not lie in the negative cone of any $a_{i}$. Assume to the contrary that $x$ lies in the negative cone of $a_{1}$. Then $\angle a_{2} a_{1} x$ or $\angle a_{3} a_{1} x$ is at least $\pi / 2$, say $\angle a_{2} a_{1} x$. So $\angle a_{2} a_{1} y \geq \pi / 2$ too. This implies that $\left|a_{2} y\right|^{2} \geq\left|a_{1} y\right|^{2}+\left|a_{1} a_{2}\right|^{2}$. Since $\tau_{v v_{i}} \leq \tau_{*}$ for all $v_{i}$, we have $\left|a_{2} y\right| \leq \tau_{*} d_{v_{2}}\left(v_{2}, z\right)=\tau_{*} d_{v_{1}}\left(v_{1}, z\right),\left|a_{1} y\right| \geq$ $d_{v_{1}}\left(v_{1}, z\right) / \tau_{*}$, and $\left|a_{1} a_{2}\right| \geq d_{v_{1}}\left(v_{1}, v_{2}\right) / \tau_{*}$. It follows that $\tau_{*}^{4} d_{v_{1}}\left(v_{1}, z\right)^{2} \geq d_{v_{1}}\left(v_{1}, z\right)^{2}+d_{v_{1}}\left(v_{1}, v_{2}\right)^{2}$. But then $d_{v_{1}}\left(v_{1}, z\right) \geq d_{v_{1}}\left(v_{1}, v_{2}\right) / \sqrt{\tau_{*}^{4}-1}=8 d_{v_{1}}\left(v_{1}, v_{2}\right)>$ $4 \rho \cdot d_{v_{1}}\left(v_{1}, v_{2}\right)$, contradicting our assumptions.

By our claim, there exists a vertex $a_{i}$ such that $a_{i} x$ stabs $a_{1} a_{2} a_{3}$, say $a_{2} x$. Let $r=d_{v_{i}}\left(v_{i}, z\right)$ for $1 \leq i \leq 3$. The following inequalities will be useful later:

$$
\begin{aligned}
& \left|a_{i} y\right| \leq \tau_{*} r . \\
& \left|a_{i} y\right| \geq r / \tau_{*} . \\
& \left|a_{i} a_{j}\right| \geq d_{v_{i}}\left(v_{i}, v_{j}\right) / \tau_{*} \geq r /\left(4 \tau_{*} \rho\right) .
\end{aligned}
$$

Note that $\tau_{*}+1 / \tau_{*}>1 /\left(4 \tau_{*} \rho\right)$ and $1 /\left(4 \tau_{*} \rho\right)+$ $1 / \tau_{*}>\tau_{*}$. This implies that one can form a triangle $b_{y} b_{i} b_{j}$ such that $\left|b_{i} b_{y}\right|=r / \tau_{*},\left|b_{i} b_{j}\right|=r /\left(4 \tau_{*} \rho\right)$, and $\left|b_{j} b_{y}\right|=\tau_{*} r$. Since $b_{j} b_{y}$ is the longest edge in the triangle $b_{y} b_{i} b_{j}$, both $\angle b_{i} b_{y} b_{j}$ and $\angle b_{i} b_{j} b_{y}$ are acute. Since $\left|b_{i} b_{y}\right| \leq\left|a_{i} y\right|,\left|b_{i} b_{j}\right| \leq\left|a_{i} a_{j}\right|$ and $\left|b_{j} b_{y}\right| \geq\left|a_{j} y\right|$, by scaling $\left|b_{j} b_{y}\right|$ down to $\left|a_{j} y\right|$ and considering the circumcircle of $b_{y} b_{i} b_{j}$, one can show that $\angle b_{y} b_{i} b_{j} \geq$ $\angle y a_{i} a_{j}$. Applying the law of cosines yields

$$
\cos \angle b_{y} b_{i} b_{j}=\frac{1-16\left(\tau_{*}^{4}-1\right) \rho^{2}}{64 \tau_{*}^{2} \rho^{2}} \cdot 8 \tau_{*}^{2} \rho .
$$

By substituting $\rho \leq \sqrt{2}$, we get $\cos \angle b_{y} b_{i} b_{j} \geq 8 \chi \tau_{*}^{2} \rho$. Thus $\angle b_{y} b_{i} b_{j}$ is acute and so is $\angle y a_{i} a_{j}$. It follows that $\angle y a_{i} a_{j} \leq \cos ^{-1}\left(8 \chi \tau_{*}^{2} \rho\right)$. If $\angle x a_{i} a_{j} \geq \pi / 2$, then $\angle y a_{i} a_{j}$ would be at least $\pi / 2$ too, which is impossible. Thus 
$\angle x a_{i} a_{j}<\pi / 2$ and so

$$
\angle x a_{i} a_{j} \leq \angle y a_{i} a_{j} \leq \cos ^{-1}\left(8 \chi \tau_{*}^{2} \rho\right) .
$$

Recall that $a_{2} x$ stabs $a_{1} a_{2} a_{3}$. The support line of $a_{2} x$ cuts $a_{1} a_{3}$ at a point $c$. Without loss of generality, assume that $\angle a_{2} c a_{3} \leq \pi / 2$. Then $\angle a_{2} a_{3} a_{1} \geq \pi / 2-$ $\angle x a_{2} a_{3} \geq \pi / 2-\cos ^{-1}\left(8 \chi \tau_{*}^{2} \rho\right)=\sin ^{-1}\left(8 \chi \tau_{*}^{2} \rho\right)$. In order to lower bound $\angle a_{3} a_{1} a_{2}$ and $\angle a_{1} a_{2} a_{3}$, there are two cases to consider depending on the acuteness of $\angle a_{2} a_{3} a_{1}$. First, for any $i$ and $j,\left|a_{i} a_{j}\right| \leq d_{v}\left(v_{i}, z\right)+$ $d_{v}\left(v_{j}, z\right) \leq \tau_{*} d_{v_{i}}\left(v_{i}, z\right)+\tau_{*} d_{v_{j}}\left(v_{j}, z\right)=2 \tau_{*} r$.

Suppose that $\angle a_{2} a_{3} a_{1} \leq \pi / 2$. By the law of sines, $\sin \angle a_{3} a_{1} a_{2}=\left(\left|a_{2} a_{3}\right| \sin \angle a_{2} a_{3} a_{1}\right) /\left|a_{1} a_{2}\right|$. Thus $\sin \angle a_{3} a_{1} a_{2} \geq \frac{r}{4 \tau_{*} \rho} \cdot 8 \chi \tau_{*}^{2} \rho \cdot \frac{1}{2 \tau_{*} r}=\chi$. Thus, $\angle a_{3} a_{1} a_{2} \geq$ $\sin ^{-1}(\chi)$. Similarly, $\angle a_{1} a_{2} a_{3} \geq \sin ^{-1}(\chi)$.

Suppose that $\angle a_{2} a_{3} a_{1}>\pi / 2$. In this case, $x$ must lie inside the triangle $a_{1} a_{2} a_{3}$. Otherwise, $\angle a_{2} a_{3} a_{1} \leq \angle x a_{3} a_{2}$ which has been proved to be acute, a contradiction. Since $x$ lies inside the triangle $a_{1} a_{2} a_{3}, \angle a_{2} a_{3} a_{1} \leq \angle x a_{3} a_{2}+\angle x a_{3} a_{1} \leq 2 \cos ^{-1}\left(8 \chi \tau_{*}^{2} \rho\right)$. Since $\angle a_{2} a_{3} a_{1}>\pi / 2, \sin \angle a_{2} a_{3} a_{1}$ is minimized when $\angle a_{2} a_{3} a_{1}$ is maximized. Thus, by the law of sines, $\sin \angle a_{3} a_{1} a_{2} \geq \frac{r}{4 \tau_{*} \rho} \cdot \sin \left(2 \cos ^{-1}\left(8 \chi \tau_{*}^{2} \rho\right)\right) \cdot \frac{1}{2 \tau_{*} r}=$ $2 \chi \sin \left(\cos ^{-1}\left(8 \chi \tau_{*}^{2} \rho\right)\right)$. By our choices of $\tau_{*}, \chi$ and $\rho, \sin \left(\cos ^{-1}\left(8 \chi \tau_{*}^{2} \rho\right)\right)>1 / 2$. So $\sin \angle a_{3} a_{1} a_{2} \geq \chi$, implying that $\angle a_{3} a_{1} a_{2} \geq \sin ^{-1}(\chi)$. Similarly, $\angle a_{1} a_{2} a_{3} \geq \sin ^{-1}(\chi)$.

From now on, we reserve $\rho$ to express the bound on $d_{v_{i}}\left(v_{i}, z\right) / \operatorname{length}(t), \sin ^{-1}(\chi)$ to express the lower bound on the angles of $\varphi_{v}(t)$, and $\rho_{1}=2 / \chi$ to express the bound on the radius-edge ratio of $\varphi_{v}(t)$, where $v, v_{i}$ and $t$ are as described in Lemma 4.1.

Lemma 4.2 below analyzes the normal of $t$ and distance of $t$ to $\Sigma$. The original proof in [3] proves (i): bounding $\angle \mathbf{n}_{v}(t), \mathbf{n}_{v}\left(v_{i}\right)$ for any point $v \in \Sigma$ nearby. Then we use Lemma 3.1 to prove (ii): transferring this angle bound to the primal space. Lastly, every point in $t$ is close to $\Sigma$ as stated in (iii).

Lemma 4.2. Define the function $\beta(c)=\sin ^{-1}(c)+$ $\sin ^{-1}\left(2 \sin \left(2 \sin ^{-1}(c)\right) / \sqrt{3}\right)$. Let $\rho \in(1, \sqrt{2}]$ and $\rho_{1}=$ $2 / \chi$ be two constants. Let $t=v_{1} v_{2} v_{3}$ be a triangle with vertices on $\Sigma$. Assume that $d_{v_{i}}\left(v_{i}, z\right) \leq 4 \rho \cdot \operatorname{length}(t)$ for some point $z \in V_{t}\left(\left\{v_{1}, v_{2}, v_{3}\right\}\right)$. For sufficiently small constant $c$, the following hold:

(i) if $v$ is a point on $\Sigma$ with $d_{v}\left(v, v_{i}\right) \leq c \gamma$ for $1 \leq$ $i \leq 3$, then for $1 \leq i \leq 3, \angle \mathbf{n}_{v}(t), \mathbf{n}_{v}\left(v_{i}\right) \leq$ $\beta\left(2 \rho_{1} c\right)+\alpha(2 c)$;

(ii) if $d_{v_{i}}\left(v_{i}, v_{j}\right) \leq c \gamma$ for $1 \leq i, j \leq 3$, then for $1 \leq i \leq 3, \angle \mathbf{n}(t), \mathbf{n}\left(v_{i}\right) \leq \beta\left(2 \rho_{1} c\right)+\alpha(2 c) ;$ (iii) if $v$ is a point on $\Sigma$ with $d_{v}\left(v, v_{i}\right) \leq c \gamma$ for $1 \leq i \leq$ 3 , then for any point $p \in t, \varphi_{v}(p)+\lambda \cdot \mathbf{n}_{v}(v) \in \varphi_{v}(\Sigma)$ and $p+\lambda^{\prime} \cdot \mathbf{n}(v) \in \Sigma$ for some $\lambda, \lambda^{\prime}$ such that $|\lambda|<40 \rho_{1}^{2} c^{2} \gamma$ and $\left|\lambda^{\prime}\right|<40 \rho_{1}^{2} c \gamma$.

To lower bound the dihedral angle between two triangles $t_{1}=v_{1} v_{3} v_{4}$ and $t_{2}=v_{2} v_{3} v_{4}$ sharing the edge $v_{3} v_{4}$, our approach is to prove that the dihedral angle between $\varphi_{v_{3}}\left(t_{1}\right)$ and $\varphi_{v_{3}}\left(t_{2}\right)$ is large in the $\varphi_{v_{3}}$-space. Then we transfer this bound to the primal space using Lemma 4.2(ii). We can do this only when $t_{1}$ and $t_{2}$ are sufficiently small. So we introduce some parameters to control the size of triangles. Let $\omega \in\left(0, \sqrt{1-1 / \tau_{*}}\right)$ be a parameter. Define the following:

- $\tau_{\omega}=1 /\left(1-\omega^{2}\right)$,

- $\forall x \in \Sigma^{*}, c_{\omega, x}=\max \left\{\lambda \leq 1 / 2: \forall y \in \Sigma^{*}, d_{x}(x, y) \leq\right.$ $\left.3 \lambda \gamma \Rightarrow \tau_{x y} \leq \tau_{\omega}\right\}$

- $c_{\omega}=\min \left\{\omega^{3}, \min _{x \in \Sigma^{*}} c_{\omega, x}\right\}$.

The parameter $\tau_{\omega}$ is less than $\tau_{*}$ and $\tau_{\omega}$ approaches 1 as $\omega$ decreases. This reflects a tigher allowance for metric distortion. Then $c_{\omega, x} \gamma$ measures the neighborhood size of a point $x \in \Sigma^{*}$ in which the metric distortion is at most $\tau_{\omega}$. Finally, we will use the global minimum $c_{\omega} \gamma$ to control the triangle size. As $\omega$ decreases, $c_{\omega} \gamma$ approaches zero. In defining $c_{\omega}$, we take a minimization with $\omega^{3}$ and this will be useful in the proof of Lemma 5.1.

The dihedral angle bound between $\varphi_{v_{3}}\left(t_{1}\right)$ and $\varphi_{v_{3}}\left(t_{2}\right)$ is obtained by showing that the tangents to the dual Voronoi edges of $\varphi_{v_{3}}\left(t_{1}\right)$ and $\varphi_{v_{3}}\left(t_{2}\right)$ are nearly orthogonal to $\varphi_{v_{3}}(\Sigma)$ around $\varphi_{v_{3}}\left(v_{3}\right)$, and the two dual Voronoi edges intersect the planes of $\varphi_{v_{3}}\left(t_{1}\right)$ and $\varphi_{v_{3}}\left(t_{2}\right)$. The exact statements are given in Lemma 4.3 below. Its proof is omitted.

LEMMA 4.3. Let $\rho=(1, \sqrt{2}]$ and $\rho_{1}=2 / \chi$ be two constants. Let $t=v_{1} v_{2} v_{3}$ be a triangle with vertices on $\Sigma$. Assume that $d_{v_{i}}\left(v_{i}, z\right) \leq c_{\omega} \gamma$ and $d_{v_{i}}\left(v_{i}, z\right) \leq$ $\rho \cdot \operatorname{length}(t)$ for some point $z \in V_{t}\left(\left\{v_{1}, v_{2}, v_{3}\right\}\right) \cap \Sigma$. Let $B_{i}$ be the ball centered at $\varphi_{v_{i}}(z)$ in the $\varphi_{v_{i}}$-space with radius $3 \cdot \operatorname{length}(t)$. Then the following hold for sufficiently small $\omega$.

(i) For all point $y \in V_{t}\left(\left\{v_{1}, v_{2}, v_{3}\right\}\right) \cap \varphi_{v_{i}}^{-1}\left(B_{i}\right)$, the acute angle between $\mathbf{n}_{v_{i}}(t)$ and the tangent to $\varphi_{v_{i}}\left(V_{t}\left(\left\{v_{1}, v_{2}, v_{3}\right\}\right)\right)$ at $\varphi_{v_{i}}(y)$ is $O(\omega)$.

(ii) Define the function $\psi(c)=2 \tan \left(\sin ^{-1}(c / 2)+\right.$ $\left.\beta\left(6 \rho_{1} c\right)+\alpha(6 c)\right)$. Let $H$ be the plane of $t$. $V_{t}\left(\left\{v_{1}, v_{2}, v_{3}\right\}\right) \cap \varphi_{v_{i}}^{-1}\left(B_{i}\right)$ contains a curve segment $\eta$ that contains $z$ in its interior and intersects $H$ exactly once at an endpoint. For any point $y \in \eta$, $d_{v_{i}}(y, z) \leq \psi\left(c_{\omega}\right) \cdot d_{v_{i}}\left(v_{i}, z\right)$. 
Using Lemma 4.3, we can orient $\varphi_{v_{3}}\left(t_{1}\right)$ and $\varphi_{v_{3}}\left(t_{2}\right)$ in anti-clockwise order, when viewed from infinity in direction $\mathbf{n}_{v_{3}}\left(v_{3}\right)$, such that $\varphi_{v_{3}}\left(v_{3} v_{4}\right)$ receive opposite orientations from $\varphi_{v_{3}}\left(t_{1}\right)$ and $\varphi_{v_{3}}\left(t_{2}\right)$. This allows us to argue that $\varphi_{v_{3}}\left(t_{1}\right)$ and $\varphi_{v_{3}}\left(t_{2}\right)$ lie on opposite sides of a plane $\varphi_{v_{3}}(G)$ through $\varphi_{v_{3}}\left(v_{3} v_{4}\right)$ and parallel to $\mathbf{n}_{v_{3}}\left(v_{3}\right)$. Then the dihedral angle bound for $\varphi_{v_{3}}\left(t_{1}\right)$ and $\varphi_{v_{3}}\left(t_{2}\right)$ follows by applying Lemma 4.2(i). When we go back to the primal space, $t_{1}$ and $t_{2}$ also lie on opposite sides of $G$ and the dihedral angle bound between $t_{1}$ and $t_{2}$ follows by Lemma 4.2(ii). The exact statements are given in the next lemma. Its proof is omitted.

Lemma 4.4. Let $\rho \in(1, \sqrt{2}]$ and $\rho_{1}=2 / \chi$ be two constants. Let $P$ be a set of vertices on $\Sigma$. Let $t_{1}=$ $v_{1} v_{3} v_{4}$ and $t_{2}=v_{2} v_{3} v_{4}$ be two adjacent triangles where $v_{i} \in P$. Assume that for $1 \leq j \leq 2, V_{t_{j}}(P) \cap \Sigma$ is a single point $z_{j}, V_{v_{3} v_{4}}(P) \cap \Sigma$ contains only one curve joining $z_{1}$ and $z_{2}$, and for any vertex $v$ of $t_{j}, d_{v}\left(v, z_{j}\right) \leq c_{\omega} \gamma$ and $d_{v}\left(v, z_{j}\right) \leq \rho \cdot \operatorname{length}\left(t_{j}\right)$. Then for sufficiently small $\omega$, the dihedral angle between $\varphi_{v_{3}}\left(t_{1}\right)$ and $\varphi_{v_{3}}\left(t_{2}\right)$ as well as the dihedral angle between $t_{1}$ and $t_{2}$ are at least $\pi-2 \beta\left(4 \rho_{1} c_{\omega}\right)-2 \alpha\left(4 c_{\omega}\right)$.

\section{Topological results}

Consider the function that maps a point away from the medial axis of $\Sigma$ to its nearest point on $\Sigma$. Let $\mu$ be the restriction of this function to Mesh $P$. The main results in this section are given in Theorem 5.1: $\mu$ is a homeomorphism between Mesh $P$ and $\Sigma$ under suitable conditions. We will need a lower bound on the interpoint distances. Define

$$
\epsilon_{\omega}=1000 \tau_{\omega}^{2} \rho_{1}^{2} \omega c_{\omega} .
$$

The lower bound on interpoint distances is $\epsilon_{\omega} \gamma$. We first show in Lemma 5.1 below that $\mu$ is injective on the vertices in $P$

Lemma 5.1. Let $\rho \in(1, \sqrt{2}]$ be a constant. Let $P$ be a set of points on $\Sigma$. There exists a value $\omega_{0}$ such that for any point $v \in P, \mu^{-1}(v)=\{v\}$, provided that the following conditions hold for some $\omega \leq \omega_{0}$.

- For any points $a, b \in P, d_{a}(a, b) \geq \epsilon_{\omega} \gamma$.

- For any triangle $t=v_{1} v_{2} v_{3}$ in Mesh $P, d_{v_{i}}\left(v_{i}, z\right) \leq$ $\rho \cdot \operatorname{length}(t)$ and $d_{v_{i}}\left(v_{i}, z\right) \leq c_{\omega} \gamma$ for some point $z \in V_{t}(P) \cap \Sigma$.

Proof. (Sketch) Let $m$ be the center of a medial ball $M$ in the primal space that touches $\Sigma$ at $v$. We go to the $\varphi_{v}$-space. Note that $\varphi_{v}(v m)$ lies on the normal line through $\varphi_{v}(v)$. Take a medial ball $M^{\prime}$ of $\varphi_{v}(\Sigma)$ at $\varphi_{v}(v)$ that intersects $\varphi_{v}(v m)$. Let $\varphi_{v}\left(m^{\prime}\right)$ be the center of $M^{\prime}$. Since the scaling factors satisfy the relation $k_{v, 1} \leq k_{v, 2} \leq k_{v, 3}$, when we go back to the primal space, $\varphi_{v}^{-1}\left(M^{\prime}\right)$ is an empty ellipsoid centered at $m^{\prime}$ and it contains a ball centered at $m^{\prime}$ with radius $\left\|m^{\prime}-v\right\|$. We conclude that $\left\|m^{\prime}-v\right\| \leq\|m-v\|$, i.e., $m^{\prime}$ lies on $m v$.

It suffices to show that no triangle in Mesh $P$ intersects $v m$. Assume to the contrary that a triangle $t=v_{1} v_{2} v_{3}$ intersects $v m$. Let $x$ be the point $t \cap v m$.

We argue that $\tau_{v v_{i}} \leq \tau_{\omega}$ for any vertex $v_{i}$ of $t$. Starting with $d_{v_{i}}\left(v_{i}, v_{j}\right) \leq d_{v_{i}}\left(v_{i}, z\right)+d_{v_{i}}\left(v_{j}, z\right)$, we get

$$
d_{v_{i}}\left(v_{i}, v_{j}\right) \leq d_{v_{i}}\left(v_{i}, z\right)+\tau_{\omega}^{2} d_{v_{j}}\left(v_{j}, z\right)<3 c_{\omega} \gamma .
$$

Since $x \in t$, there exists $j$ such that $d_{v_{i}}\left(v_{i}, x\right) \leq$ $d_{v_{i}}\left(v_{i}, v_{j}\right)<3 c_{\omega} \gamma$. So $x \in \Sigma^{*}$ and $\tau_{x v_{i}} \leq \tau_{\omega}$ by the definition of $c_{\omega}$. By definition, $F_{v}=F_{x}$ and so $\tau_{v v_{i}}=\tau_{x v_{i}} \leq \tau_{\omega}$.

We claim that $x$ lies between $m^{\prime}$ and $v$. Otherwise, in the primal space, since the distance from $x$ to $\Sigma$ is at least the distance from $x$ to the boundary of the medial ball $M$ centered at $m$, this distance is at least $\left\|m^{\prime}-v\right\|=d_{v}\left(m^{\prime}, v\right) / \sqrt{k_{v, 3}} \geq \gamma / \sqrt{k_{v, 3}}$. But if we apply Lemma 4.2(iii) using any vertex of $t$, we can conclude that $\|x-v\|=O\left(c_{\omega} \gamma\right)$ which is less than $\gamma / \sqrt{k_{v, 3}}$ for sufficiently small $\omega$, a contradiction. This proves our claim. Hence, $\varphi_{v}(x)$ lies between $\varphi_{v}\left(m^{\prime}\right)$ and $\varphi_{v}(v)$.

We go back to the $\varphi_{v}$-space. Since $M^{\prime}$ is the medial ball of $\varphi_{v}(\Sigma), M^{\prime}$ does not contain any vertex of $\varphi_{v}(t)$. By Lemma 4.3(ii), $V_{t}\left(\left\{v_{1}, v_{2}, v_{3}\right\}\right)$ intersects the plane of $t$ at a point $q$ such that

$$
d_{v_{i}}\left(v_{i}, q\right) \leq\left(1+\psi\left(c_{\omega}\right)\right) d_{v_{i}}\left(v_{i}, z\right)<2 c_{\omega} \gamma .
$$

Take the smallest enclosing ball $B$ of $\varphi_{v}(t)$ centered at $\varphi_{v}(q)$ in the $\varphi_{v}$-space. So the radius of this ball is $\max _{1 \leq i \leq 3} d_{v}\left(v_{i}, q\right) \leq \tau_{\omega} \cdot \max _{1 \leq i \leq 3} d_{v_{i}}\left(v_{i}, q\right)<2 \tau_{\omega} c_{\omega} \gamma$.

First, we consider the case in which $B$ does not contain $\varphi_{v}(v)$. Since the diameter of $B$ is no more than $4 \tau_{\omega} c_{\omega} \gamma<\gamma, B$ does not intersect the medial axis of $\varphi_{v}(\Sigma)$ and so $\varphi_{v}\left(m^{\prime}\right)$ lies outside $B^{\prime}$. Thus the segment $\varphi_{v}\left(m^{\prime} v\right)$ and $\varphi_{v}(t)$ lie on opposite sides of the bisector plane of $B$ and $M^{\prime}$. But then $\varphi_{v}(t)$ cannot intersect $\varphi_{v}\left(m^{\prime} v\right)$ at $\varphi_{v}(x)$, a contradiction.

Second, we consider the case in which $B$ contains $\varphi_{v}(v)$. So $d_{v}\left(v, v_{i}\right) \leq 4 \tau_{\omega} c_{\omega} \gamma$. In the rest of the proof, we show that this leads to the contradiction that $d_{v}(v, z)<d_{v_{j}}\left(v_{j}, z\right)$ for some $j$. Lemma 4.2(iii) implies that $d_{v}(x, v)<640 \tau_{\omega}^{2} \rho_{1}^{2} c_{\omega}^{2} \gamma$. For $1 \leq i \leq 3$, since $d_{v}\left(v, v_{i}\right) \geq \epsilon_{\omega} \gamma$, we get $d_{v}(x, v) \leq 640 \tau_{\omega}^{2} \rho_{1}^{2}$. $\left(c_{\omega}^{2} / \epsilon_{\omega}\right) \cdot d_{v}\left(v, v_{j}\right)$. By definition, $\epsilon_{\omega}=1000 \tau_{\omega}^{2} \rho_{1}^{2} \omega c_{\omega}$ and $c_{\omega} \leq \omega^{3}$, so we obtain

$$
d_{v}(x, v)<\omega^{2} \cdot d_{v}\left(v, v_{i}\right) .
$$

Next, we bound the distance $d_{v}(x, q)$. First, for any $v_{i}$,

$$
d_{v}\left(v_{i}, x\right) \geq d_{v}\left(v, v_{i}\right)-d_{v}(v, x) \geq\left(1-\omega^{2}\right) \cdot d_{v}\left(v, v_{i}\right) .
$$


Observe that, as $\varphi_{v}(x)$ lies inside $\varphi_{v}(t)$, there is always a vertex $v_{j}$ such that $\varphi_{v}\left(\angle q x v_{j}\right) \geq \pi / 2$. Therefore,

$$
\begin{aligned}
d_{v}(x, q) & \leq \sqrt{d_{v}\left(v_{j}, q\right)^{2}-d_{v}\left(v_{j}, x\right)^{2}} \\
& \leq \sqrt{\tau_{\omega}^{2} d_{v_{j}}\left(v_{j}, q\right)^{2}-\left(1-\omega^{2}\right)^{2} d_{v}\left(v, v_{j}\right)^{2}}
\end{aligned}
$$

Since $d_{v_{j}}\left(v_{j}, q\right) \leq 2 c_{\omega} \gamma$ by equation (5.2) and $d_{v}\left(v, v_{j}\right) \geq \epsilon_{\omega} \gamma$ by assumption, we have $d_{v}\left(v, v_{j}\right) \geq$ $\epsilon_{\omega} /\left(2 c_{\omega}\right) \cdot d_{v_{j}}\left(v_{j}, q\right)$. Using $\lambda$ to denote $1000 \tau_{\omega}^{2} \rho_{1}^{2}$, we get $d_{v}\left(v, v_{j}\right) \geq(\lambda \omega / 2) \cdot d_{v_{j}}\left(v_{j}, q\right)>\tau_{\omega} \lambda \omega /(2 \sqrt{2}) \cdot d_{v_{j}}\left(v_{j}, q\right)$. Thus,

$$
d_{v}(x, q) \leq \tau_{\omega} d_{v_{j}}\left(v_{j}, q\right) \cdot \sqrt{1-\left(1-\omega^{2}\right)^{2} \cdot\left(\lambda^{2} \omega^{2} / 8\right)}
$$

For sufficiently small $\omega$, this simplifies to

$$
d_{v}(x, q) \leq \tau_{\omega}\left(1-\lambda^{2} \omega^{2} / 32\right) \cdot d_{v_{j}}\left(v_{j}, q\right)
$$

Since $\lambda=1000 \tau_{\omega}^{2} \rho_{1}^{2}, \lambda^{2} \omega^{2} / 64 \geq 4 \omega^{3}$. Also, since $\psi\left(c_{\omega}\right)=O\left(c_{\omega}\right)=O\left(\omega^{3}\right), \lambda^{2} \omega^{2} / 64 \geq 2 \psi\left(c_{\omega}\right)$ for sufficiently small $\omega$. Hence, we obtain

$$
d_{v}(x, q) \leq \tau_{\omega}\left(1-4 \omega^{2}-2 \psi\left(c_{\omega}\right)\right) \cdot d_{v_{j}}\left(v_{j}, q\right) .
$$

Recall that the radius of $B$ is equal to $d_{v}\left(v_{k}, q\right)$ for some $v_{k}$. By equation $(5.3), d_{v}(v, x) \leq \omega^{2} \cdot d_{v}\left(v, v_{i}\right)$ for any vertex $v_{i}$. Thus $d_{v}(v, x) \leq \omega^{2} \cdot\left(d_{v}(v, q)+d_{v}\left(v_{i}, q\right)\right) \leq$ $2 \omega^{2} \cdot \operatorname{radius}(B)=2 \omega^{2} \cdot d_{v}\left(v_{k}, q\right) \leq 2 \tau_{\omega} \omega^{2} \cdot d_{v_{k}}\left(v_{k}, q\right)=$ $2 \tau_{\omega} \omega^{2} \cdot d_{v_{j}}\left(v_{j}, q\right)$. It follows that

$$
\begin{aligned}
d_{v}(v, q) & \leq d_{v}(v, x)+d_{v}(x, q) \\
& \leq \tau_{\omega}\left(1-2 \omega^{2}-2 \psi\left(c_{\omega}\right)\right) \cdot d_{v_{j}}\left(v_{j}, q\right) .
\end{aligned}
$$

By Lemma $4.3(\mathrm{ii}), d_{v_{j}}(q, z) \leq \psi\left(c_{\omega}\right) \cdot d_{v_{j}}\left(v_{j}, z\right)$. So $d_{v}(q, z) \leq \tau_{\omega} \psi\left(c_{\omega}\right) \cdot d_{v_{j}}\left(v_{j}, z\right)$. Hence,

$$
\begin{aligned}
d_{v}(v, z) & \leq d_{v}(v, q)+d_{v}(q, z) \\
& <\tau_{\omega}\left(1-2 \omega^{2}\right) \cdot d_{v_{j}}\left(v_{j}, z\right) .
\end{aligned}
$$

Since $\tau_{\omega}=1 /\left(1-\omega^{2}\right), \tau_{\omega}\left(1-2 \omega^{2}\right)<1$. So $d_{v}(v, z)<d_{v_{j}}\left(v_{j}, z\right)$ which contradicts the fact that $z \in V_{t}(P)$.

In the next result, when we say $\operatorname{Mesh} P$ is a 2manifold combinatorially, we mean that the incidence relations among the triangles indicate a 2 -manifold, but we do not care whether Mesh $P$ self-intersects.

ThEOREM 5.1. Let $\rho \in(1, \sqrt{2}]$ be a constant. Let $P$ be a set of points on $\Sigma$. There exists a value $\omega_{0}$ such that Mesh $P$ does not self-intersect and Mesh $P$ is homeomorphic to $\mu($ Mesh $P)$, provided that the following conditions hold for some $\omega \leq \omega_{0}$.

- Mesh $P$ is combinatorially a 2-manifold.
- For each triangle $t$ in Mesh $P, V_{t}(P) \cap \Sigma$ is a single point. For each edge e in Mesh $P, V_{e}(P) \cap \Sigma$ is a simple open curve.

- For any points $a, b \in P, d_{a}(a, b) \geq \epsilon_{\omega} \gamma$. For any triangle $t=v_{1} v_{2} v_{3}$ in $\operatorname{Mesh} P, d_{v_{i}}\left(v_{i}, z\right) \leq \rho$. length $(t)$ and $d_{v_{i}}\left(v_{i}, z\right) \leq c_{\omega} \gamma$, where $z=V_{t}(P) \cap \Sigma$.

Hence, under the above conditions, if Mesh $P$ contains a vertex in each connected component of $\Sigma$, then Mesh $P$ is homeomorphic to $\Sigma$.

Proof. (Sketch) Let $\omega_{0}$ be a small enough value for which the previous results hold. By our assumption, it can be checked that Mesh $P$ avoids the medial axis of $\Sigma$. We show that $\mu$ is a homeomorphism between Mesh $P$ and $\mu($ Mesh $P)$. Since Mesh $P$ is compact and $\mu$ is continuous, $\mu(\operatorname{Mesh} P)$ is also compact. Thus it suffices to show that $\mu$ is injective.

First, we show that $\mu$ is injective on each triangle $t$ in Mesh $P$. Assume to the contrary that there is a point $x \in \Sigma$ such that $\mu$ maps two distinct points $p$ and $q$ in a triangle $t$ to $x$. So $p, q$ and $x$ lie on the line normal to $\Sigma$ at $x$. Let $v$ be a vertex of $t$. Let $z$ be the point $V_{t}(P) \cap \Sigma$. For any other vertex $w$ of $t, d_{v}(v, w) \leq$ $d_{v}(v, z)+\tau_{*} d_{w}(w, z)=O\left(c_{\omega} \gamma\right)$. By Lemma $4.2(\mathrm{iii})$, the distance from $p$ to $\Sigma$ is $O\left(c_{\omega} \gamma\right)$. Thus $\|p-x\|=O\left(c_{\omega} \gamma\right)$. So for sufficiently small $\omega, p \in \Sigma^{*}$ and so $F_{p}$ is defined and $F_{p}=F_{x}$ by definition. Since $\|p-x\|=O\left(c_{\omega} \gamma\right)$, $d_{p}(p, x)=d_{x}(p, x)=\sqrt{k_{x, 3}} \cdot\|p-x\|=O\left(\sqrt{k_{x, 3}} \cdot c_{\omega} \gamma\right)=$ $O\left(\sqrt{c_{\omega}} \gamma\right)$ for sufficiently small $\omega$. Since $p \in t$, there is a vertex $w$ of $t$ such that $d_{v}(v, p) \leq d_{v}(v, w)$ which has been shown to be $O\left(c_{\omega} \gamma\right)$. We conclude that $d_{v}(v, x) \leq$ $d_{v}(v, p)+d_{v}(p, x) \leq O\left(c_{\omega} \gamma\right)+\tau_{*} d_{p}(p, x)=O\left(\sqrt{c_{\omega}} \gamma\right)$. Вy Lemma 3.2(ii), $\mathbf{n}(v)$ and $\mathbf{n}(x)$ are nearly parallel. Then by Lemma $4.2(\mathrm{ii}), t$ and $\mathbf{n}(x)$ are nearly orthogonal. But since $p q$ lies on $t, t$ must be parallel to $\mathbf{n}(x)$, a contradiction.

Second, we show that $\mu$ is injective. For each point $x \in \mu(\operatorname{Mesh} P)$, define $h(x)$ to be the number of points mapped to $x$ by $\mu$. One can visualize $h(x)$ as the number of intersection points between Mesh $P$ and the line segment $s_{x}$ joining the centers of the two medial balls at $x$. Since $\mu$ is injective on each triangle, $h(x)$ is a finite number and as $x$ varies over $\Sigma, h(x)$ stays constant if $s_{x}$ does not cross any triangle edge. Suppose that $h(x)$ changes when $s_{x}$ crosses a triangle edge. Since Mesh $P$ is combinatorially a 2-manifold, this edge is incident to two triangles $t_{1}$ and $t_{2}$. The change in $h(x)$ implies that $\mu\left(t_{1}\right)$ and $\mu\left(t_{2}\right)$ overlap. But this contradicts the large dihedral angle between $t_{1}$ and $t_{2}$ (Lemma 4.4) and the fact that $t_{1}$ and $t_{2}$ are almost perpendicular to the normal of any common vertex (Lemma 4.2(ii)). Thus $h$ is a constant function. 
Then Lemma 5.1 implies that $h=1$. It follows that $\mu$ is a homeomorphism.

\section{Anisotropic meshing algorithm}

We first discuss some primitives to be used by our algorithm AnisoMesh. Given a sample set $P$, we assume an incremental algorithm to compute $\operatorname{Vor} P$, e.g. the incremental lower envelope algorithm in [9]. The lower envelope can be used to answer which Voronoi edge, facet, or cell contains a point $x$ in $\mathbb{R}^{3}$. The critical points of a curve or surface with respect to a direction $d$ are the points at which $d$ is normal to the curve or surface. We use $E: \mathbb{R}^{3} \rightarrow \mathbb{R}$ to denote the implicit function for $\Sigma$, i.e., $x \in \Sigma$ iff $E(x)=0$. For any $v, w \in P$, let $H_{v w}(x)=0$ denote the equation of $V_{v w}(\{v, w\})$. We assume a numerical solver for a system of equations. For example, we can then compute the intersection points between $\Sigma$ and a Voronoi edge of Vor $P$. Anisomesh uses the following three routines. Let $x_{1}, x_{2}$, and $x_{3}$ be the coordinate axes. Given any equation $G(x)=0$, by taking partial derivatives, we get $G^{\prime}(x)=\left(\frac{\partial G(x)}{\partial x_{1}}, \frac{\partial G(x)}{\partial x_{2}}, \frac{\partial G(x)}{\partial x_{3}}\right)$, which is the equation of a normal at $x$ to $G(x)$.

- FacetTest(edge $v_{1} v_{2}$ in Mesh $P$ ): Compute the vector $d=\mathbf{n}\left(v_{1}\right) \times\left(v_{2}-v_{1}\right)$. If $V_{v_{1} v_{2}}(P) \cap \Sigma$ contains any critical point with respect to $d$ or if $V_{v_{1} v_{2}}(P)$ is tangent to $\Sigma$ at some point, return one as follows. The intersection $V_{v_{1} v_{2}}\left(\left\{v_{1}, v_{2}\right\}\right) \cap \Sigma$ is a collection of disjoint smooth closed curves. The equation of the tangent at any point $x \in V_{v_{1} v_{2}}\left(\left\{v_{1}, v_{2}\right\}\right) \cap \Sigma$ is $H_{v_{1} v_{2}}^{\prime}(x) \times E^{\prime}(x)$. The set of critical points on $V_{v_{1} v_{2}}\left(\left\{v_{1}, v_{2}\right\}\right) \cap \Sigma$ with respect to $d$ is defined by: $E(x)=0, H_{v_{1} v_{2}}(x)=0,\left\langle H_{v_{1} v_{2}}^{\prime}(x) \times E^{\prime}(x), d\right\rangle=$ 0 . Solve it and return any solution that lies on $V_{v_{1} v_{2}}(P)$. The tangency case is handled similarly. At the contact point $x, H_{v_{1} v_{2}}^{\prime}(x) \times E^{\prime}(x)=0$. Thus, solve the system: $E(x)=0, H_{v_{1} v_{2}}(x)=$ $0, H_{v_{1} v_{2}}^{\prime}(x) \times E^{\prime}(x)=0$, and return any solution that lies on $V_{v_{1} v_{2}}(P)$.

- PatchMax (vertex $v$ in Mesh $P)$ : Return the point $x$ in $V_{v}(P) \cap \Sigma$ that maximizes $d_{v}(v, x)$ as follows. We first handle points on the boundary of $V_{v}(P) \cap \Sigma$. The intersection points on the edges of $V_{v}(P)$ can be computed directly. Take a Voronoi neighbor $w$ of $v$. If the maximum is achieved at some interior point $x \in V_{v w}(P) \cap \Sigma$, the tangent to $\varphi_{v}\left(V_{v w}(P) \cap\right.$ $\Sigma)$ at $\varphi_{v}(x)$ is orthogonal to the vector $\varphi_{v}(x-v)$. The equation of the tangent to $\varphi_{v}\left(V_{v w}(P) \cap \Sigma\right)$ at $\varphi_{v}(x)$ is $F_{v} \cdot\left(H_{v w}^{\prime}(x) \times E^{\prime}(x)\right)$. Solve the system: $E(x)=0, H_{v w}(x)=0,\left\langle F_{v} \cdot(x-v), F_{v} \cdot\left(H_{v w}^{\prime}(x) \times\right.\right.$ $\left.\left.E^{\prime}(x)\right)\right\rangle=0$. Collect the solutions that lie on
$V_{v w}(P)$ and repeat for other Voronoi neighbors of $v$. If the maximum is achieved at some interior point $x \in V_{v}(P) \cap \Sigma, \mathbf{n}_{v}(x)$ makes an angle 0 or $\pi$ with the vector $\varphi_{v}(x-v)$. The equation of the normal to $\varphi_{v}(\Sigma)$ at $\varphi_{v}(x)$ is $F_{v}^{-1} \cdot E^{\prime}(x)$. Solve the system: $E(x)=0,\left(F_{v} \cdot(x-v)\right) \times\left(F_{v}^{-1} \cdot E^{\prime}(x)\right)=0$. Collect the solutions that lie inside $V_{v}(P)$. After collecting all the candidate points, return the point $x$ that maximizes $d_{v}(v, x)$.

- SilhTest(vertex $v$ in Mesh $P$ ): If the set $\{x \in$ $\left.V_{v}(P) \cap \Sigma: \angle \mathbf{n}(x), \mathbf{n}(v)=\pi / 2\right\}$ is non-empty, return a point in it as follows. The larger set $J=\{x \in \Sigma: \angle \mathbf{n}(x), \mathbf{n}(v)=\pi / 2\}$ is a collection of disjoint smooth closed curves [14]. The set $J$ satisfies the system: $E(x)=0, K(x)=0$, where $K(x)=\left\langle E^{\prime}(x), \mathbf{n}(v)\right\rangle$. We check whether $J$ intersects the boundary of $V_{v}(P)$ by solving the system: $E(x)=0, K(x)=0, H_{v w}(x)=0$, for all Voronoi neighbors $w$ of $v$. If there is a solution lying on $V_{v, w}(P)$, return it. Otherwise, let $d$ be any direction orthogonal to $\mathbf{n}(v)$. If some component of $J$ lies completely inside $V_{v}(P), J$ has a critical point with respect to $d$ inside $V_{v}(P)$. The tangent to $J$ at $x$ is given by $K^{\prime}(x) \times E^{\prime}(x)$. Solve the system: $E(x)=0, K(x)=0,\left\langle K^{\prime}(x) \times E^{\prime}(x), d\right\rangle=0$. If there is a solution lying inside $V_{v}(P)$, return it.

The SilhTest routine was first introduced in [11] for isotropic surface meshing. We are ready to describe our algorithm to mesh $\Sigma$.

\section{$\operatorname{AnisoMesh}(\Sigma)$ :}

1. Initialize $P$ to contain one critical point of $\Sigma$ with respect to the vertical direction. (Even though $\Sigma$ may have multiple components, one critical point is enough for initialization.)

2. Repeatedly apply a rule from the following list to insert a new point into $P$ until no rule is applicable. A rule with smaller index has higher priority. Let $\rho \in\left(\tau_{*}, \sqrt{2}\right], \zeta \in(0, \pi / 4)$ and $\ell>0$ be some constants.

(a) If there is a triangle $t=v_{1} v_{2} v_{3}$ in Mesh $P$ such that $d_{v_{i}}\left(v_{i}, x\right)>\rho \cdot \operatorname{length}(t)$ for some point $x \in$ $V_{t}(P) \cap \Sigma$, insert $x$.

(b) If a Voronoi edge $V_{v_{1} v_{2} v_{3}}(P)$ intersects $\Sigma$ at least twice or tangentially, insert the point $x \in$ $V_{v_{1} v_{2} v_{3}}(P) \cap \Sigma$ that maximizes $d_{v_{i}}\left(v_{i}, x\right)$.

(c) If FacetTest $\left(v_{1} v_{2}\right)$ returns a point $x$ for some edge $v_{1} v_{2}$ in Mesh $P$, insert $x$.

(d) If the incident triangles of a vertex $v$ in Mesh $P$ do not form a disk combinatorially, insert the point $x$ 
returned by $\operatorname{PatchMax}(v)$. (By not being a disk combinatorially, we mean the incidence relations among the incident triangles do not indicate a topological disk, and we do not care whether some triangles cross each other.)

(e) If Mesh $P$ self-intersects, find the triangle $t=$ $v_{1} v_{2} v_{3}$ in Mesh $P$ such that $d_{v_{i}}\left(v_{i}, x\right)$ is maximum, where $x=V_{t}(P) \cap \Sigma$, and insert $x$.

(f) If $\operatorname{SilhTest}(v)$ returns a point $x$ for some vertex $v$ of Mesh $P$, insert $x$.

(g) If there is a triangle $t=v_{1} v_{2} v_{3}$ in Mesh $P$ such that $\angle \mathbf{n}_{v_{i}}(t), \mathbf{n}_{v_{i}}\left(v_{i}\right)$ or $\angle \mathbf{n}(t), \mathbf{n}\left(v_{i}\right)$ exceeds $\zeta$ for some $1 \leq i \leq 3$, insert the point $V_{t}(P) \cap \Sigma$.

(h) If $d_{v_{1}}\left(v_{1}, v_{2}\right)>\ell$ for some edge $v_{1} v_{2}$ in Mesh $P$, take a triangle $t$ incident to $v_{1} v_{2}$ and insert the point $V_{t}(P) \cap \Sigma$.

Except for the upper bound on triangle size, all conditions in Theorem 5.1 are enforced in steps 2(a)(e). Step 2(c) is a sufficient condition to ensure that each Voronoi facet does not intersect in a closed curve. Then step 2(d) ensures that each Voronoi facet intersects $\Sigma$ in a simple open curve, and the boundary of $V_{v}(P) \cap \Sigma$ is a single closed curve for each $v \in P$. After step 2(e), tunnels or isolated components in $V_{v}(P) \cap \Sigma$ may still exist and step 2(f) detects that. Previous experience with the isotropic surface meshing problem [11] suggests that even if the triangulation is homeomorphic to $\Sigma$, it may not be a good geometric approximation of $\Sigma$. Therefore, steps $2(\mathrm{~g})$ and $2(\mathrm{~h})$ are introduced to enforce user-supplied upper bounds on on the maximum angle deviation and maximum edge length. The termination of Anisomesh follows from establishing a lower bound on the interpoint distances throughout the algorithm.

LeMma 6.1. Let $\omega<1 /\left(1000 \tau_{*}^{3} \rho_{1}^{2}\right)$ be a small enough constant such that Theorem 5.1 holds, $\beta\left(6 \rho_{1} \tau_{*} \epsilon_{\omega}\right)+$ $\alpha\left(6 \tau_{*} \epsilon_{\omega}\right)<\zeta$, and $3 \tau_{*} \epsilon_{\omega} \gamma<\ell$. After each point insertion in ANISOMESH, $d_{a}(a, b) \geq \epsilon_{\omega} \gamma$ for any vertices $a, b \in P$.

Proof. (Sketch) The lemma is true after step (1). We prove the lemma inductively. It suffices to show that $d_{v}(v, x) \geq \tau_{*} \epsilon_{\omega} \gamma$ if a point $x \in V_{v}(P) \cap \Sigma$ is inserted. Then $d_{x}(w, x) \geq \epsilon_{\omega} \gamma$ for all $w \in P$; otherwise, $d_{w}(w, x) \leq \tau_{*} d_{x}(w, x)<\tau_{*} \epsilon_{\omega} \gamma \leq d_{v}(v, x)$, a contradiction.

If step 2(a) inserts a point $x \in V_{t}(P) \cap \Sigma$, then for any vertex $v_{i}$ of $t, d_{v_{i}}\left(v_{i}, x\right)>\rho \cdot \operatorname{length}(t) \geq \rho \epsilon_{\omega} \gamma>$ $\tau_{*} \epsilon_{\omega} \gamma$ as $\rho>\tau_{*}$.

Suppose that step 2(b) inserts a point in $V_{t}(P) \cap \Sigma$, where $t=v_{1} v_{2} v_{3}$. Assume to the contrary that $d_{v_{i}}\left(v_{i}, z\right)<\tau_{*} \epsilon_{\omega} \gamma$ for all points $z \in V_{t}(P) \cap \Sigma$. Suppose that $V_{t}(P)$ intersects $\Sigma$ at two points $z_{1}$ and $z_{2}$. The case where $V_{t}(P)$ meets $\Sigma$ tangentially is easier. We go to the $\varphi_{v_{1}}$-space. Let $B_{1}$ be the ball centered at $\varphi_{v_{1}}\left(z_{1}\right)$ in the $\varphi_{v_{1}}$-space with radius $3 \cdot \operatorname{length}(t) \geq 3 \epsilon_{\omega} \gamma$. Let $B$ and $B^{\prime}$ be the two medial balls of $\varphi_{v_{1}}(\Sigma)$ meeting at $\varphi_{v_{1}}\left(z_{1}\right)$. By Lemma 3.2(ii) and Lemma 4.2(i), $\mathbf{n}_{v}\left(z_{1}\right)$ and $\mathbf{n}_{v}(t)$ are nearly parallel. Then by Lemma $4.3(\mathrm{i})$, $\varphi_{v}\left(V_{t}(P)\right) \cap B_{1}$ lies close to the normal at $\varphi_{v_{1}}\left(z_{1}\right)$. So $\varphi_{v}\left(V_{t}(P)\right) \cap B_{1}$ lies inside $B \cup B^{\prime}$, which implies that $d_{v_{1}}\left(z_{1}, z_{2}\right) \geq \operatorname{radius}\left(B_{1}\right) \geq 3 \epsilon_{\omega} \gamma$, contradicting $d_{v_{1}}\left(z_{1}, z_{2}\right) \leq d_{v_{1}}\left(v_{1}, z_{1}\right)+d_{v_{1}}\left(v_{1}, z_{2}\right)<2 \tau_{*} \epsilon_{\omega} \gamma$.

Suppose that FacetTest $\left(v_{1} v_{2}\right)$ returns a point $x$ in step 2(c). We skip the tangency case as the analysis is similar. So some plane $H$ orthogonal to $\mathbf{n}\left(v_{1}\right) \times\left(v_{2}-v_{1}\right)$ meets $V_{v_{1} v_{2}}(P) \cap \Sigma$ tangentially at $x$. Then $\varphi_{v_{1}}(H)$ is orthogonal to $\mathbf{n}_{v_{1}}\left(v_{1}\right) \times \varphi_{v_{1}}\left(v_{2}-v_{1}\right)$ and it meets $\varphi_{v_{1}}\left(V_{v_{1} v_{2}}(P) \cap \Sigma\right)$ tangentially at $\varphi_{v_{1}}(x)$. Assume to the contrary that $d_{v_{1}}\left(v_{1}, x\right)<\tau_{*} \epsilon_{\omega} \gamma$. We go to the $\varphi_{v_{1}}$-space. It can be checked that $d_{v_{1}}\left(v_{1}, v_{2}\right)<3 \tau_{*} \epsilon_{\omega} \gamma$. Let $e_{x}$ be a normal to $\varphi_{v_{1}}\left(V_{v_{1} v_{2}}(P)\right)$ at $\varphi_{v_{1}}(x)$ pointing towards the side of $\varphi_{v_{1}}\left(v_{2}\right)$. Using an analysis in proving Lemma 4.3(i), one can show that $e_{x}$ is nearly parallel to $\varphi_{v_{1}}\left(v_{2}-v_{1}\right)$. By Lemma 3.2(ii), $\mathbf{n}_{v_{1}}(x)$ is nearly parallel to $\mathbf{n}_{v_{1}}\left(v_{1}\right)$. By Lemma 3.2(i), the vector $\varphi_{v_{1}}\left(v_{2}-v_{1}\right)$ is nearly orthogonal to $\mathbf{n}_{v_{1}}\left(v_{1}\right)$. So $\mathbf{n}_{v_{1}}(x) \times e_{x}$ is nearly parallel to $\mathbf{n}_{v_{1}}\left(v_{1}\right) \times \varphi_{v_{1}}\left(v_{2}-v_{1}\right)$, which is normal to $\varphi_{v_{1}}(H)$. But since $\mathbf{n}_{v_{1}}(x) \times e_{x}$ is tangent to $\varphi_{v_{1}}\left(V_{v_{1} v_{2}}(P) \cap \Sigma\right)$ at $\varphi_{v_{1}}(x), \mathbf{n}_{v_{1}}(x) \times e_{x}$ is parallel to $\varphi_{v_{1}}(H)$, a contradiction.

We omit the analysis of step 2(d). Suppose that step 2(e) inserts the point $x=V_{t}(P) \cap \Sigma$ for some triangle $t=v_{1} v_{2} v_{3}$. Before the insertion of $x$, Mesh $P$ satisfies all the conditions in Theorem 5.1 except the upper bound on triangle size. Thus $d_{v_{i}}\left(v_{i}, x\right)>c_{\omega} \gamma=$ $\epsilon_{\omega} \gamma /\left(1000 \tau_{\omega}^{2} \rho_{1}^{2} \omega\right)>\tau_{*} \epsilon_{\omega} \gamma$

Suppose that step $2(\mathrm{f})$ inserts the point $x \in$ $V_{v}(P)$. So $\angle \mathbf{n}(v), \mathbf{n}(x)=\pi / 2$ which implies that $\angle \mathbf{n}_{v}(v), \mathbf{n}_{v}(x)=\pi / 2$. Thus $d_{v}(v, x)$ must be greater than $\tau_{*} \epsilon_{\omega} \gamma$ by Lemma 3.2(ii).

Suppose that step $2(\mathrm{~g})$ inserts the point $x=$ $V_{t}(P) \cap \Sigma$ for $t=v_{1} v_{2} v_{3}$. So $\angle \mathbf{n}_{v_{i}}(t), \mathbf{n}_{v_{i}}\left(v_{i}\right)$ or $\angle \mathbf{n}(t), \mathbf{n}\left(v_{i}\right)$ exceeds $\zeta$ for some $i$. If $d_{v_{i}}\left(v_{i}, x\right)<$ $\tau_{*} \epsilon_{\omega} \gamma$, it can be checked that $d_{v_{i}}\left(v_{i}, v_{j}\right)<3 \tau_{*} \epsilon_{\omega} \gamma$. But then Lemma 4.2(i) and (ii) imply that both $\angle \mathbf{n}_{v_{i}}(t), \mathbf{n}_{v_{i}}\left(v_{i}\right)$ and $\angle \mathbf{n}(t), \mathbf{n}\left(v_{i}\right)$ are no more than $\beta\left(6 \rho_{1} \tau_{*} \epsilon_{\omega}\right)+\alpha\left(6 \tau_{*} \epsilon_{\omega}\right)<\zeta$, a contradiction.

Suppose that step 2(h) finds an edge $v w$ such that $d_{v}(v, w)>\ell$. Let $z$ be the point $V_{t}(P) \cap \Sigma$ for some triangle $t$ incident to $v w$. Then $d_{v}(v, z) \geq \tau_{*} \epsilon_{\omega} \gamma$; otherwise, $d_{v}(v, w) \leq d_{v}(v, z)+d_{v}(z, w) \leq d_{v}(v, z)+\tau_{*}^{2} d_{w}(w, z)<$ $3 \tau_{*} \epsilon_{\omega} \gamma<\ell$, a contradiction. 
TheOrem 6.1. Let $\rho \in\left(\tau_{*}, \sqrt{2}\right], \ell>0, \zeta \in(0, \pi / 4)$, and $\chi=\frac{1-32\left(\tau_{*}^{4}-1\right)}{128 \tau_{*}^{2}}$ be some constants. Assume that $\Sigma$ is a smooth closed implicit surface and there is an oracle to return the 2D metric tensor at any point $x \in \Sigma$. $\operatorname{Anisomesh}(\Sigma)$ samples a set $P$ of vertices on $\Sigma$ and computes a triangulation Mesh $P$ homeomorphic to $\Sigma$. For any triangle $t$ in Mesh $P$ and for any edge $v w$ of $t$, $d_{v}(v, w) \leq \ell, \angle \mathbf{n}(t), \mathbf{n}(v) \leq \zeta, \angle \mathbf{n}_{v}(t), \mathbf{n}_{v}(v) \leq \zeta$, and each angle of $\varphi_{v}(t)$ is at least $\sin ^{-1}(\chi)$.

Proof. (Sketch) The geometric approximation is guaranteed by previous results. Let $S_{v}$ denote $V_{v}(P) \cap \Sigma$. By the end of Anisomesh, $S_{v}$ is connected and $\partial S_{v}$ is a single closed curve. Then it has been shown that $S_{v}$ is a topological disk and it projects to a topological disk on the tangent plane at $v[11]$. We refine $\partial S_{v}$ by inserting a vertex in the interior of each boundary edge $V_{v w}(P) \cap \Sigma$ of $S_{v}$. Then we refine Mesh $P$ into a triangulation $T$ by refining each triangle using a barycentric subdivision. Thanks to the barycentric subdivision, for each vertex $v \in P$, there is a bijection between the edges in its link in $T$ (i.e., the boundary of the fan of triangles incident to $v$ ) and the refined $\partial S_{v}$. Afterwards, this boundary homeomorphism can be extended to a homeomorphism between the fan of triangles incident to $v$ in $T$ and $S_{v}$. Stitching these homeomorphisms together yields a homeomorphism between $\Sigma$ and Mesh $P$.

\section{Conclusion}

There are several directions for future work. We plan to conduct an experimental study of the effectiveness of the algorithm. There has been some recent work on remeshing a given surface mesh in the anisotropic setting, e.g. [1], and this problem awaits a thorough theoretical study. This has been done in the isotropic case by Dey, Li, and Ray [13]. The problem of generating a 3D anisotropic volume mesh remains open.

\section{References}

[1] P. Alliez, D. Cohen-Steiner, O. Devillers, B. Levy, and M. Desbrun. Anisotropic polygonal remeshing. ACM Transactions on Graphics, 22 (2003), 485-493.

[2] N. Amenta and M. Bern. Surface reconstruction by Voronoi filtering. Discr. Comput. Geom., 22 (1999), 481-504.

[3] N. Amenta, S. Choi, T. K. Dey and N. Leekha. A simple algorithm for homeomorphic surface reconstruction. Internat. J. Comput. Geom. Applications, 12 (2002), 125-141.

[4] J. Bloomenthal. Polygonization of implicit surfaces. Proc. Comput. Aided Geom. Design, 5 (1988), 341-355.
[5] J.-D. Boissonnat and F. Cazals. Natural neighbor coordinates of points on a surface. Comput. Geom. Theory Appl., 19 (2001), 87-120.

[6] J.-D. Boissonnat and S. Oudot. Provably good surface sampling and approximation. Eurographics Sympos. Geom. Process. (2003), 9-18.

[7] J.-D. Boissonnat, D. Cohen-Steiner and G. Vegter. Isotopic implicit surface meshing. Proc. 36th ACM Sympos. Theory Comput., 2004, 301-309.

[8] J.-D. Boissonnat, C. Wormser, and M. Yvinec. Anisotropic diagrams: Labelle Shewchuk approach revisited. CCCG 2005.

[9] B. Chazelle, H. Edelsbrunner, L. Guibas, and M. Sharir. A singlyexponential stratification scheme for real semi-algebraic varieties and its applications. Theoret. Comput. Sci., 84 (1991), 77-105.

[10] H.-L. Cheng, T. K. Dey, H. Edelsbrunner and J. Sullivan. Dynamic skin triangulation. Discrete Comput. Geom., 25 (2001), 525-568.

[11] S.-W. Cheng, T. K. Dey, E. A. Ramos, and T. Ray. Sampling and meshing a surface with guaranteed topology and geometry. Proc. 20th Annu. Sympos. Comput. Geom., 2004, 280-289.

[12] J.C. Cuiliere. An adaptive method for the automatic triangulation of 3D parametric surfaces. Computer Aided Design, 30 (1998), 139-149.

[13] T.K. Dey, G. Li, and T. Ray. Polygonal surface remeshing with Delaunay refinement. Proc. 14th International Meshing Roundable, 2005, 343-351.

[14] H. Edelsbrunner and J. Harer. Jacobi sets of multiple Morse functions. In Foundations of Computational Mathematics, eds. F. Cucker, R. DeVore, P. Olver and E. Sueli, Cambridge Univ. Press, 2003, 37-57.

[15] T. Karkanis and A.J. Stewart. Curvature-dependent triangulation of implicit surfaces. IEEE Computer Graphics and Applications, 21 (2001), 60-69.

[16] F. Labelle and J.R. Shewchuk. Anisotropic Voronoi diagrams and guaranteed-quality anisotropic mesh generation. Proc. 19th Annu. Sympos. Comput. Geom., 2003, 191-200.

[17] T.S. Lau and S.H. Lo. Finite element mesh generation over analytical surfaces. Computers and Structures, 59 (1996), 301-309.

[18] G. Leibon and D. Letscher. Delaunay triangulations and Voronoi diagrams for Riemannian manifolds. Proc. 16th Annu. Sympos. Comput. Geom., 2000, 341-349.

[19] W.E. Lorensen and H.E. Cline. Marching cubes: a high resolution 3D surface reconstruction algorithm. Computer Graphics (SIGGRAPH '87 Proceedings), 21 (1987), 163-170.

[20] M. Sharir. Almost tight bounds for lower envelopes in higher dimensions. Discrete \& Comput. Geom., 12 (1994), 327-345.

[21] J.R. Tristano, S.J. Owen, and S.A. Canann. Advancing front surface mesh generation in parametric space using a Riemannian surface definition. Proc. 7th Intl. Meshing Roundtable 1998. 